\title{
Appendices
}

Appendix A: Combination of Search terms

Intervention terms

Outcome terms

\begin{tabular}{cc}
\hline "early warning" & "maternal mortality" (MeSH) \\
"early detection" & "maternal morbidity" (MeSH) \\
"track and trigger" & "obstet* complication" \\
"monitoring chart" & "obstet* haemorrhage" \\
"vital\$ chart" & "matern* outcome" \\
EWS & "matern* sepsis" \\
MOEWS & "eclampsia" \\
MEOWS & "chorioamnionitis" \\
\hline
\end{tabular}

Appendix B1: Quality assessment tool

\begin{tabular}{|c|c|c|}
\hline Domain & Signalling questions & Scoring \\
\hline Patient selection & Was a continuous or random sample of participants & Yes \\
\hline \multirow[t]{5}{*}{ Risk of bias } & enrolled? & No \\
\hline & & Unclear \\
\hline & Did the study avoid unnecessary exclusions? & Yes \\
\hline & & No \\
\hline & & Unclear \\
\hline Applicability & Are there concerns that the included participants do & Yes \\
\hline \multirow[t]{2}{*}{ concern } & not match the review questions? & No \\
\hline & & Unclear \\
\hline Index test & Were the reference range adjusted for physiological & Yes \\
\hline \multirow[t]{3}{*}{ Risk of bias } & change in pregnancy? & No \\
\hline & & Unclear \\
\hline & Was the trigger threshold prespecified? & Yes \\
\hline
\end{tabular}


Applicability Are there concerns that the index test, its conduct or Yes concern interpretation differ from review questions? No

$\begin{array}{lll}\begin{array}{l}\text { Reference } \\ \text { standard }\end{array} & \text { Is the index test compared to a reference standard } & \text { Yes } \\ \text { Risk of bias } & & \text { No } \\ & \text { Were the reference standard results interpreted } & \text { Yes } \\ & \text { without knowledge of the results of the index test } & \text { No } \\ \text { Applicability } & \text { Are there concerns that the target condition as } & \text { Yes } \\ \text { concern } & \text { defined by the reference standard does not match } & \text { No } \\ \text { the review questions? } & \text { Unclear } \\ \text { Flow of } & \text { Is the attrition rate acceptable }(<20 \%) \text { ? } & \text { Yes } \\ \text { participants } & & \text { No } \\ \text { Risk of bias } & & \text { Unclear } \\ \text { Applicability } & \text { Could the drop out participants or missing data be } & \text { Yes } \\ \text { concern } & \text { systematically similar to those who completed? } & \text { No }\end{array}$

Unclear

Appendix B2: QUADAS-2 Scoring guideline (order of answers not important)

\section{Risk of bias}

Score

Question 1

Question 2

\begin{tabular}{ccc}
\hline Low & Yes & Yes \\
& Yes & Unclear \\
High & No & No \\
& No & Unclear \\
\hline
\end{tabular}




\begin{tabular}{ccc}
\hline Unclear & Yes & No \\
& Unclear & Unclear \\
\hline Low & Concern about applicability \\
\hline High & Yes \\
Unclear & No \\
\hline
\end{tabular}

\begin{tabular}{|c|c|c|c|c|c|c|c|}
\hline \multicolumn{8}{|c|}{ Appendix C: Quality of individual studies } \\
\hline \multirow[t]{2}{*}{ Study } & \multicolumn{4}{|c|}{ RISK OF BIAS } & \multicolumn{3}{|c|}{ CONCERN ABOUT APPLICABILITY } \\
\hline & $\begin{array}{l}\text { Patient } \\
\text { selection }\end{array}$ & $\begin{array}{l}\text { Index } \\
\text { test }\end{array}$ & $\begin{array}{l}\text { Reference } \\
\text { standard }\end{array}$ & $\begin{array}{l}\text { Participants } \\
\text { flow }\end{array}$ & $\begin{array}{l}\text { Patient } \\
\text { selection }\end{array}$ & $\begin{array}{l}\text { Index } \\
\text { test }\end{array}$ & $\begin{array}{l}\text { Reference } \\
\text { standard }\end{array}$ \\
\hline $\begin{array}{l}\text { Singh S et al, } \\
2012\end{array}$ & $\bar{L}$ & $\bar{L}$ & $\mathrm{H}$ & $\bar{L}$ & $\bar{L}$ & $\bar{L}$ & $\bar{L}$ \\
\hline $\begin{array}{l}\text { Carle C et al, } \\
2013\end{array}$ & $\mathrm{~L}$ & $\mathrm{~L}$ & $\mathrm{~L}$ & $\mathrm{U}$ & $\mathrm{L}$ & $\mathrm{L}$ & $\mathrm{U}$ \\
\hline $\begin{array}{l}\text { Singh A et al, } \\
2016\end{array}$ & $\mathrm{~L}$ & $\mathrm{~L}$ & $\mathrm{H}$ & $\mathrm{L}$ & $\mathrm{L}$ & $\mathrm{L}$ & $\mathrm{U}$ \\
\hline $\begin{array}{l}\text { Hedriana HL et } \\
\text { al, } 2016\end{array}$ & $U$ & $\mathrm{~L}$ & $\mathrm{U}$ & $\mathrm{L}$ & $\mathrm{L}$ & $\mathrm{L}$ & $\mathrm{H}$ \\
\hline $\begin{array}{l}\text { Ryan HM et al, } \\
2017\end{array}$ & L & L & $\mathrm{H}$ & L & L & L & L \\
\hline $\begin{array}{l}\text { Paternino- } \\
\text { Caicedo et al, } \\
2017\end{array}$ & $\mathrm{U}$ & $\mathrm{L}$ & $\mathrm{L}$ & $\mathrm{L}$ & $\mathrm{L}$ & $\mathrm{L}$ & $\mathrm{L}$ \\
\hline $\begin{array}{l}\text { Edwards ES et } \\
\text { al, } 2015\end{array}$ & $U$ & $L$ & $L$ & $\mathrm{H}$ & $L$ & $L$ & $L$ \\
\hline $\begin{array}{l}\text { Lappen RJ et al, } \\
2010\end{array}$ & $U$ & $U$ & $L$ & $\mathrm{H}$ & $L$ & $\mathrm{H}$ & $U$ \\
\hline $\begin{array}{l}\text { Von-Dadelszen P } \\
\text { et al, } 2011\end{array}$ & $L$ & $L$ & $U$ & $\mathrm{~L}$ & $L$ & $\mathrm{H}$ & $U$ \\
\hline Payne BA et al, & $\mathrm{L}$ & $\mathrm{L}$ & $U$ & $\mathrm{~L}$ & $\mathrm{~L}$ & $\mathrm{H}$ & $U$ \\
\hline
\end{tabular}


Nathan HL et al, 2017

Austin DM et al,

\begin{tabular}{lllllll}
\hline$H$ & L & U & L & L & U & U
\end{tabular}

2013

Maguire PJ et al,

\begin{tabular}{lllllll}
\hline$L$ & $L$ & $U$ & U & L & L & L
\end{tabular}

Karen AP 2015

Maguire PJ, Amy

\begin{tabular}{llllllll}
\hline$L$ & $L$ & U & H & L & L & U
\end{tabular}

C.O et al, 2015

Shields E L et al

\begin{tabular}{lllllllll}
\hline L & L & U & H & L & H & L & & L
\end{tabular}

2016

Sheikh $\mathrm{S}$ et al,

\begin{tabular}{lllllll}
\hline $\mathrm{L}$ & $\mathrm{H}$ & $\mathrm{U}$ & $\mathrm{L}$ & $\mathrm{H}$ & $\mathrm{H}$ & $\mathrm{U}$
\end{tabular}

2017

Merriel A et al,

\begin{tabular}{lllllll}
\hline U & $\mathrm{L}$ & $\mathrm{U}$ & $\mathrm{L}$ & $\mathrm{L}$ & $\mathrm{L}$ & $\mathrm{U}$
\end{tabular}

2017

$\mathrm{L}=$ Low, $\mathrm{H}=$ High, $\mathrm{U}=$ Unclear 